\title{
RESEARCH
}

Open Access

\section{Global research mapping of substance use disorder and treatment 1971-2017: implications for priority setting}

Bach Xuan Tran ${ }^{1,8^{*}}$ (D) Mackenzie Moir ${ }^{2,4}$, Carl A. Latkin ${ }^{8,9}$, Brian J. Hall ${ }^{8,9}$, Cuong Tat Nguyen ${ }^{3}$, Giang Hai Ha ${ }^{3}$, Nam Ba Nguyen ${ }^{4}$, Cyrus S. H. Ho ${ }^{6}$ and Roger C. M. Ho ${ }^{5,7}$

\begin{abstract}
Background: Globally, substance use disorders are prevalent and remain an intractable public health problem for health care systems. This study aims to provide a global picture of substance use disorders research.

Methods: The Web of Science platform was used to perform a cross-sectional analysis of scientific articles on substance use disorders and treatment. Characteristics of publication volume, impact, growth, authors, institutions, countries, and journals were examined using descriptive analysis and network visualization graphs.

Results: Thirteen thousand six hundred eighty-five papers related to illicit drugs (5403), tobacco (4469), and alcohol (2137) use disorders and treatment were published between 1971 and 2017. The number of publications on Mindfulness and Digital medicine topics had the highest increase with more than 300\% since 2003-2007 despite later presence than other methods. The number of papers on other non-pharmaceutical therapies (behavioral therapy, cognitive behavioral therapy, skills training or motivational interviewing) grew gradually, however, the growth rate was lower every 5 -year period. The United States is the substance use disorder research hub of the world with the highest volume of publications (8232 or $60.2 \%$ ) and total citations (252,935 or $65.2 \%$ ), number of prolific authors ( 25 of top 30 or $83 \%$ ) and institutions (24 of top 26 or 92\%), formed the most international research partnerships (with 96 distinct countries). The international collaboration followed a pattern based on geographic proximity and cultural similarity.

Conclusions: This study offers a comprehensive picture of the global trend of publications of substance use disorder. Findings suggest a need for research policy that supports the examination of interventions that culturally adhere to different local contexts to address substance use disorder in communities.
\end{abstract}

Keywords: Bibliometric analysis, Substance use disorder, Global, mapping, Substance abuse treatment, Alcohol, Smoking

\section{Background}

Substance use and misuse are globally prevalent and remain an ongoing health crisis affecting every region of the world. In 2016, the United Nations Office on Drugs and Crime (UNODC) estimated that 275 million people aged 15-64 used drugs at least once [1], and the prevalence of

\footnotetext{
* Correspondence: bach.jhu@gmail.com

'Institute for Preventive Medicine and Public Health, Hanoi Medical University, Hanoi, Vietnam

${ }^{8}$ Bloomberg School of Public Health, Johns Hopkins University, Baltimore, MD, USA

Full list of author information is available at the end of the article
}

drug use and drug use disorders has increased significantly in the period 2010-2016 [2]. Likewise, global alcohol consumption rates per capita had slightly increased over a 15-year period [3]. Regarding tobacco smoking, in 2015, $20.2 \%$ of the world's population aged $>15$ years old were current smokers [4]. As a result, substance use disorders caused approximately 20 million disability-adjusted life years (DALYs) and 8.6 million years of life lost (YLL) across regions and countries [5-7]. The World Health Organization (WHO) estimated that in 2016, drug and alcohol use disorders were responsible for respectively 160,235 and 145,565 deaths, which increased markedly

(C) The Author(s). 2019 Open Access This article is distributed under the terms of the Creative Commons Attribution 4.0 International License (http://creativecommons.org/licenses/by/4.0/), which permits unrestricted use, distribution, and 
from $1990[8,9]$. These ongoing burdens are becoming great challenges for health systems of every country [2].

In the past decades, treatments of substance use disorders, both non-pharmacological and pharmacological methods, have been well-documented [10]. In terms of tobacco use, along with nicotine replacement therapies, non-pharmacological treatments such as "counseling", "self-help", and "behavioral therapies" have been proven as effective therapies that can maintain smoking cessation more than 6-month follow up $[11,12]$. Similarly, non-pharmacological approaches including "skills training", "cognitive behavior therapy" and "family and couple therapy" are used widely to improve alcohol dependence [13]. For drug use disorder, substitutional treatments including methadone, buprenorphine or naltrexone maintenance treatment are used commonly as replacement therapies, along with traditional medicine and motivational enhancement therapies [14].

Prevention and management of substance use disorder have been considered one of the top priorities. Internationally, the WHO and the United Nations have been at the forefront with many efforts in synthesizing evidence and developing guidelines and frameworks to combat this public health crisis [15-18]. There has also been an increasing focus on developing a standard set of indicators, which allow to build an optimal monitoring system for harm reduction interventions [19]. Despite these efforts, gaps in research, training, treatment, service delivery, and capacity building related to substance use disorder are recognized, with greater negative impacts on low-to-middle income countries [20]. Moreover, these challenges vary across regions and countries significantly. This heterogeneity requires contextually sensitive approaches to the development and implementation of 'locally' compatible policy solutions. This study attempts to 'take stock' of the currently available substance use literature through the use of bibliometric methods [21]. In literature, few studies using this method to investigate the current status and tendency of research publications in substance misuse in Saudi Arabia [22] or drug/cocaine addiction only [23-25]. This work aims to provide clinicians, policymakers and other stakeholders a better understanding of 1) the trend and the current focus of international research efforts regarding substance use disorders; 2) what evidence is currently available on related subjects; and 3) where research, evidence and service gaps remain ongoing challenges.

\section{Methods}

We used the Web of Science (WoS) to retrieve research publications focusing on substance use, comorbidity, treatment, and interventions. The WoS outweights other databases such as Scopus or MEDLINE. Initial, the WoS allows to extract information based on research disciplines that could not be done in other databases. Second, the WoS database comprises of leading and high impact scientific journals, while other databases included journals with a variety of quality [26-28]. Third, the WoS has a comprehensive coverage of scientific publications from 1900 until now with a diversity of research disciplines. Forth, the WoS has advantages in allowing to perform advanced search tool, refine the results to particular criteria, and evaluate the research productivity. This database offers a wide range of information about title, authors, keywords, sources/organizations, countries, languages, total citations as well as the average citation per literature. Our analysis focused on substance use disorder articles published from January 1st, 1971 to December 31st, 2017 in peer-reviewed journals. We did not include grey literature, conference proceedings, or books/book chapters in our analysis. Articles written in any language other than English were excluded.

\section{Search strategy}

Our search strategy was performed according to following steps:

\section{Inclusion step}

The literature from the WoS was retrieved using a set of search terms, focusing on 1) substance use disorder (including all illicit substances), 2) tobacco use, and 3) alcohol use disorder [29]. Synonyms for each search term were identified by research team including senior researchers (B.X.T and G.H.H) and junior researchers (C.T.N and N.B.N), whom had experiences in the field of substance use disorder. The keywords used were referred from previously published systematic reviews [30-33]. Our search query is outlined in Table 1.

\section{Exclusion step}

We excluded articles which were 1) published later than 31 December 2017; 2) documents that were not articles such as book chapters and conference proceedings; 3) without author details; and 4) written in any language other than English. We also used the WOS database functionality to exclude publications in unrelated fields of study [see Additional file 1].

\section{Data extraction}

Data were exported from the WoS in text format and imported into Microsoft Excel for analysis. Exported data included: (1) Total number of publications by year for three types of substance use disorder (Illicit drugs, tobacco, and alcohol); (2) Name and details of journals; (3) Authors' name, affiliation and number of publications; (4) Top cited articles; (5) Types of articles; (6) Title of the paper; (7) Year of publication; (8) Author's 
Table 1 Search Query Text

First, we searched for the three main kinds of substance use disorder

(1) smoking OR tobacco-smoking OR nicotine OR tobacco-usedisorder* OR Cigar* OR Tobacco

(2) substance-abuse OR substance-related-disorder* OR substanceabuse-intravenous OR drug-rehabilitation OR drug-usage OR drugdepend* OR substance-use-disorder* OR opioid-related-disorder* OR opioid-abuse OR opioid-addict* OR Drug-Abuse OR Drug-Addict* OR Marijuana-abuse OR Marijuana-addict*

(3) alcohol*-drinking OR alcohol*-addiction OR alcohol*-abuse OR alcohol-rehabilitation OR alcohol-depend*

Second, we developed separate search queries for several commonly used interventions and methods for treating substance abuse disorder. These included:

(4) Behavioral therapy with six sub-fields: Cognitive behavioral ther apy, self-help, Motivational enhancement therapy, Motivational inter viewing, The Matrix Model, and 12 Step Facilitation Therapy,

(5) Psychological treatment method with three sub-fields: Family ther apy, Group counseling OR mixed counseling and Mindfulness,

(6) Pharmacological therapy with four sub-fields: Nicotine replace ment therapy and Non nicotine medication were for nicotine addic tion, for alcohol abuse were Disulfiram therapy OR Naltrexone OR Campral, and for opioid addiction we applied: Alternative-Drug* OR Methadone OR Buprenorphine OR Naltrexone, and

(7) Other treatment methods included: herbal medicine, digital medicine, telephony, and acupuncture.

In final step, we connected query 1 through 3 with the "AND" operator with queries 4 through 7 [see Additional file 1].

and WoS's keywords; (9) Number of citations of each article; and (10) Abstracts.

\section{Data analysis}

Our analysis of authorship involved an initial sorting of data based on the number of authors, total number of citations, citations per paper, h-index, and how many papers were written collaboratively [34]. We directly downloaded these data via the WOS Citation report.

We used search terms (see Table 1 (1), (2), (3)) using Microsoft Excel to quantify the volume of publications related to (1) drugs use disorder; (2) nicotine dependency; and (3) alcohol addiction. We then applied search terms (see Table 1 (4), (5), (6)) to determine the number of publications focusing on the following therapies and interventions: (4) Behavioral therapy OR Behavioral treatment, (5) Psychological treatment method, (6) Pharmacological therapy OR Pharmacological-treatment, and (7) Other treatment methods.

Next, we tracked the growth of publications in different substance use disorders. We used the period 5-year intervals across 1998 to 2017 to evaluate the "index of change" for each intervention. This measure shows a change of a research field by comparing the growth of publication of one period compared with that of previous one [35].

VOSviewer software (http://www.vosviewer.com/) was used to 1) create visualization graphs indicating contributions and collaborative efforts of sixty-one countries with at least five publications; 2) Visualizing the co-occurrence terms in titles and abstracts of all publications with at least 250 times of presence [36]. Networks highlighted the trend and provide the insight of the development of substance use disorders in our dataset at any level: node, connection, network and overall system [37].

\section{Results}

Table 2 illustrates general information of our dataset. This topic attracted the concern of research community that showed by the volume of publications increased markedly every 5 years, especially between 2013 and 2017 (one-third of all articles). Nearly 70\% of the papers in all three methods was collaborative research of 2-3 authors and 4-6 authors, that is promised a main trend year after year, reflecting the multi-investigator in a research study [38]. Substance use disorder was the research field across disciplines, that was showed by half of the publication assigning to 2 or 3 research areas. The use of alcohol and tobacco is increasing rapidly in developing countries [39], however, most of the lead author are from developed countries (China was the only Asian country in the list of top 10 country of first author).

Figure 1 reveals an acceleration of publications in the field of substance abuse disorder after 1990. The number of papers in this period accounted for approximately three-fourths of total number of publications. Noticably, the growth of publications was different among three types of substance use disorders (Table 3). The number of publications on Mindfulness and Digital medicine topics had the highest increase with more than 300\% since 2003-2007 despite later presence than other methods. The number of papers on other non-pharmaceutical therapies (behavioral therapy, cognitive behavioral therapy, skills training or motivational interviewing) grew gradually, however, the growth rate was lower every 5-year period.

Table 4 presents that the Journal of Substance Abuse and Treatment was the most common journal with the highest number of publications (613 papers), followed by the Drug and Alcohol Dependence (591) and the Addiction journal (400).

Table 5 shows information of 30 most prolific researchers. They published from 44 to 117 publications and had 1137 to 5878 citations. Among these authors, 25 (83\%) had affiliations in the American institutions, 
Table 2 General characteristics of selected articles

\begin{tabular}{|c|c|c|c|c|c|c|c|}
\hline \multirow[b]{2}{*}{ Characteristic } & \multirow[b]{2}{*}{ Category } & \multicolumn{2}{|l|}{ Tobacco } & \multicolumn{2}{|c|}{ Drug addiction } & \multicolumn{2}{|l|}{ Alcohol } \\
\hline & & Number & Percent & Number & Percent & Number & Percent \\
\hline Total number of papers & & 4469 & 100 & 5403 & 100 & 2137 & 100 \\
\hline \multirow[t]{7}{*}{ Year of publication } & 2013-2017 & 1688 & 37.8 & 2090 & 38.7 & 850 & 39.8 \\
\hline & 2008-2012 & 1302 & 29.1 & 1565 & 29.0 & 536 & 25.1 \\
\hline & 2003-2007 & 803 & 18.0 & 855 & 15.8 & 364 & 17.0 \\
\hline & 1998-2002 & 364 & 8.1 & 489 & 9.1 & 234 & 10.9 \\
\hline & 1993-1997 & 198 & 4.4 & 278 & 5.1 & 126 & 5.9 \\
\hline & 1988-1992 & 82 & 1.8 & 70 & 1.3 & 24 & 1.1 \\
\hline & $<1988$ & 32 & 0.7 & 57 & 1.0 & 3 & 0.1 \\
\hline \multirow[t]{5}{*}{ Number of authors } & 1 & 286 & 6.4 & 418 & 7.8 & 166 & 7.8 \\
\hline & $2-3$ & 1300 & 29.1 & 1691 & 31.3 & 730 & 34.2 \\
\hline & $4-6$ & 1772 & 39.7 & 2197 & 40.7 & 866 & 40.5 \\
\hline & $7-10$ & 903 & 20.2 & 905 & 16.7 & 309 & 14.5 \\
\hline & $>10$ & 208 & 4.7 & 192 & 3.6 & 66 & 3.1 \\
\hline \multirow[t]{5}{*}{ Number of subject category } & 1 & 2261 & 50.6 & 2492 & 46.1 & 970 & 45.4 \\
\hline & 2 & 1709 & 38.2 & 2331 & 43.1 & 934 & 43.7 \\
\hline & 3 & 470 & 10.5 & 557 & 10.3 & 220 & 10.3 \\
\hline & 4 & 22 & 0.5 & 14 & 0.3 & 9 & 0.4 \\
\hline & 5 & 4 & 0.1 & 8 & 0.1 & 3 & 0.1 \\
\hline \multirow{10}{*}{$\begin{array}{l}\text { Country of first author (top 10) } \\
\text { (number of papers) }\end{array}$} & 1 & USA & 2464 & USA & 3329 & USA & 1444 \\
\hline & 2 & England & 321 & Australia & 208 & Australia & 80 \\
\hline & 3 & Canada & 179 & England & 200 & England & 76 \\
\hline & 4 & Australia & 167 & Canada & 195 & Canada & 63 \\
\hline & 5 & Netherlands & 99 & Italy & 115 & Netherlands & 40 \\
\hline & 6 & Spain & 90 & Germany & 82 & Sweden & 33 \\
\hline & 7 & China & 64 & Spain & 80 & Italy & 27 \\
\hline & 8 & Italy & 60 & France & 77 & Germany & 26 \\
\hline & 9 & Germany & 57 & Netherlands & 70 & Iran & 20 \\
\hline & 10 & Switzerland & 57 & Switzerland & 61 & Switzerland & 18 \\
\hline
\end{tabular}

while other authors worked at institutions in England, Italy and Canada.

Table 6 illustrates the research productivity among top 41 countries. The top five countries were North America (the United States of America and Canada), Europe (England and Italy), and Australia. The United States of America was the substance use disorder knowledge hub in the world, and ranked at the top of all indices and was the main collaborator of 38 countries in the list. There were $70 \%$ of research conducted in the U.S. as a result of national collaborations, whereas more than $40 \%$ of research projects in other countries were the results of international cooperation.

Strength of collaborative partnerships and contributions among countries are shown in Fig. 2. There were three major geographical research clusters including: 1) England, Scotland, Wales and Turkey; 2) East, and
South-East Asia, such as The Peoples Republic of China, India, Taiwan, and Vietnam; and 3) Central and Eastern Europe, for example, Austria, Poland, and Bulgaria. This distribution might be justified by the geographical proximity and cultural similarity among countries in each cluster.

Figure 3 presents the most popular terms with at least 250 appearances drawn from the title and abstract, which could be separated into three topic clusters: 1) Intervention (Red), 2) Types of addictions: Tobacco addiction (Green), Drug addiction (Blue), Alcohol (Purple), and 3) Effects (Yellow).

\section{Intervention}

The word "Intervention" appeared the most with 9438 times appearances in papers focusing on smoking addition, followed by drug use and alcohol. Moreover, 


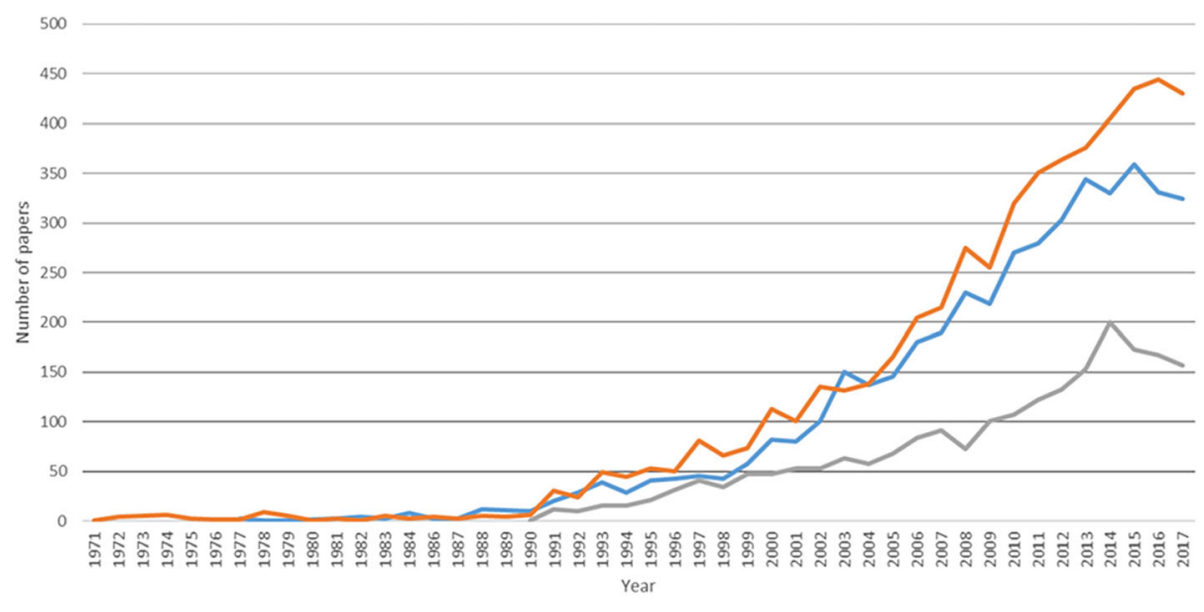

Fig. 1 Number of papers by year in publication

these papers presented intervention programs in different populations such as adolescents and students; and with a variety of methods such as training program, counseling, and motivational interviewing.

\section{Types of addiction}

"Smoker" and "smoking" frequently appeared alongside "withdrawal". The term "pharmacotherapy" appeared in papers focusing on nicotine replacement and non-nicotine replacement. Papers with titles and abstracts that used the term "drug" also used the terms "intervention", "problem", or "disorder". The term "alcohol" frequently appeared in studies on adolescents.

\section{Effect}

The term "effect" appeared 8640 times in the titles and abstracts, co-occurring with terms related to intervention/program or effects of "smoking", "alcohol" and "drug" use on human health.

\section{Discussion}

This study provided an insight of global publications trend, research productivity and collaboration networks in the field of substance use disorder and treatment. In this study, we demonstrated a rapid increase of publications in the past few decades, especially papers focusing on mindfulness and e-health interventions. This growth was led by leading institutions located in the United States of America, United Kingdom, Europe, Canada, and Australia.

The current finding suggested that the number of publications about traditional intervention approaches such as behavioral therapy, cognitive behavioral therapy, or counselling raised gradually over years. This result was in line with previous reviews that these methods were the most common way in intervening alcohol, tobacco and drug-use disorder [4042]. Moreover, we found a substantial shift toward the use of mindfulness and e-health interventions in this field. In literature, mindfulness interventions were effective in preventing alcohol relapse and decreasing withdrawal symptoms when compared with cognitivebehavioral treatment [43, 44]. Also, publications about digital medicine (or e-health) were found in all three types of substance use disorder. This intervention is delivered via computers or mobile phones, which enables to facilitate interactions between patients and clinicians. Although its long-term effects were limited, digital medicine was found to be useful when combined with human supports [45].

We found a pattern of collaborative research networks that showed a preference for geographical proximity and cultural similarity. Northern European countries (Norway, Finland, and Sweden), three countries in The United Kingdom (England, Scotland, and Wales) created a cluster in collaboration in research. Meanwhile, another cluster among the U.S., Japan and China (mainland), Taiwan (China), Vietnam, South Africa, India and Ukraine was generated. This can be explained by the strong international scientific collaboration between US, Japan and Asia countries. Moreover, health topics were major concern of those developing countries and received support from developed countries $[46,47]$. These findings suggest the importance of increasing research capacity and establish collaborative partnerships between high income and developing countries.

There are several research studies and policy related implications derived from our findings. First, our study found there was an upward trend in the 
Table 3 Number of publication and the growth of publication (\%)

\begin{tabular}{|c|c|c|c|c|c|c|c|c|c|c|c|c|c|c|c|}
\hline \multirow[b]{3}{*}{ Name of therapy } & \multicolumn{15}{|c|}{ Number of paper (Growth of publication - \%) } \\
\hline & \multicolumn{5}{|c|}{ Tobacco } & \multicolumn{5}{|l|}{ Drug } & \multicolumn{5}{|c|}{ Alcohol } \\
\hline & $\begin{array}{l}1993- \\
1997\end{array}$ & $\begin{array}{l}1998- \\
2002\end{array}$ & $\begin{array}{l}2003- \\
2007\end{array}$ & $\begin{array}{l}2008- \\
2012\end{array}$ & $\begin{array}{l}2013- \\
2017\end{array}$ & $\begin{array}{l}1993- \\
1997\end{array}$ & $\begin{array}{l}1998- \\
2002\end{array}$ & $\begin{array}{l}2003- \\
2007\end{array}$ & $\begin{array}{l}2008- \\
2012\end{array}$ & $\begin{array}{l}2013- \\
2017\end{array}$ & $\begin{array}{l}1993- \\
1997\end{array}$ & $\begin{array}{l}1998- \\
2002\end{array}$ & $\begin{array}{l}2003- \\
2007\end{array}$ & $\begin{array}{l}2008- \\
2012\end{array}$ & $\begin{array}{l}2013- \\
2017\end{array}$ \\
\hline \multicolumn{16}{|c|}{ Non pharmaceutical therapy } \\
\hline Behavioral therapy & 2 & $\begin{array}{l}5 \\
(150)\end{array}$ & $\begin{array}{l}19 \\
(280)\end{array}$ & $\begin{array}{l}44 \\
(131.6)\end{array}$ & $\begin{array}{l}72 \\
(63.6)\end{array}$ & 84 & $\begin{array}{l}187 \\
(122.6)\end{array}$ & $\begin{array}{l}326 \\
(74.3)\end{array}$ & $\begin{array}{l}620 \\
(90.2)\end{array}$ & $\begin{array}{l}876 \\
(41.3)\end{array}$ & 47 & $\begin{array}{l}106 \\
(125.5)\end{array}$ & $\begin{array}{l}157 \\
(48.1)\end{array}$ & $\begin{array}{l}242 \\
(54.1)\end{array}$ & $\begin{array}{l}453 \\
(87.2)\end{array}$ \\
\hline $\begin{array}{l}\text { Cognitive behavioral } \\
\text { therapy }\end{array}$ & 3 & $\begin{array}{l}5 \\
(66.7)\end{array}$ & $7(40)$ & $\begin{array}{l}35 \\
(400)\end{array}$ & $\begin{array}{l}50 \\
(42.9)\end{array}$ & 17 & $\begin{array}{l}63 \\
(270.6)\end{array}$ & $\begin{array}{l}125 \\
(98.4)\end{array}$ & $\begin{array}{l}259 \\
(107.2)\end{array}$ & $\begin{array}{l}496 \\
(91.5)\end{array}$ & 16 & $\begin{array}{l}46 \\
(187.5)\end{array}$ & $\begin{array}{l}69 \\
(50)\end{array}$ & $\begin{array}{l}109 \\
(58)\end{array}$ & $\begin{array}{l}258 \\
(136.7)\end{array}$ \\
\hline Skills training & 7 & $\begin{array}{l}4 \\
(-42.9)\end{array}$ & $\begin{array}{l}10 \\
(150)\end{array}$ & $\begin{array}{l}15 \\
(50)\end{array}$ & $\begin{array}{l}25 \\
(66.7)\end{array}$ & 10 & $\begin{array}{l}39 \\
(290)\end{array}$ & $\begin{array}{l}91 \\
(133.3)\end{array}$ & $\begin{array}{l}109 \\
(19.8)\end{array}$ & $\begin{array}{l}108 \\
(-0.9)\end{array}$ & 11 & $\begin{array}{l}40 \\
(263.6)\end{array}$ & $\begin{array}{l}56 \\
(40)\end{array}$ & $\begin{array}{l}78 \\
(39.3)\end{array}$ & $\begin{array}{l}80 \\
(2.6)\end{array}$ \\
\hline $\begin{array}{l}\text { Motivational } \\
\text { interviewing }\end{array}$ & 1 & $\begin{array}{l}10 \\
(900)\end{array}$ & $\begin{array}{l}22 \\
(120)\end{array}$ & $\begin{array}{l}51 \\
(131.8)\end{array}$ & $\begin{array}{l}86 \\
(68.6)\end{array}$ & 8 & $\begin{array}{l}22 \\
(175)\end{array}$ & $\begin{array}{l}73 \\
(231.8)\end{array}$ & $\begin{array}{l}172 \\
(135.6)\end{array}$ & $\begin{array}{l}226 \\
(31.4)\end{array}$ & 7 & $\begin{array}{l}22 \\
(214.3)\end{array}$ & $\begin{array}{l}54 \\
(145.5)\end{array}$ & $\begin{array}{l}102 \\
(88.9)\end{array}$ & $\begin{array}{l}158 \\
(54.9)\end{array}$ \\
\hline The Matrix Model & 0 & $0(-)$ & $0(-)$ & $0(-)$ & $0(-)$ & 0 & $1(-)$ & $\begin{array}{l}2 \\
(100)\end{array}$ & $\begin{array}{l}1 \\
(-50)\end{array}$ & $1(0)$ & 0 & $0(-)$ & $0(-)$ & $1(-)$ & $1(0)$ \\
\hline $\begin{array}{l}12 \text { Step Facilitation } \\
\text { Therapy }\end{array}$ & 0 & $0(-)$ & $1(-)$ & $\begin{array}{l}0 \\
(-100)\end{array}$ & $1(-)$ & 2 & $\begin{array}{l}7 \\
(250)\end{array}$ & $\begin{array}{l}11 \\
(57.1)\end{array}$ & $\begin{array}{l}15 \\
(36.4)\end{array}$ & $\begin{array}{l}24 \\
(60)\end{array}$ & 2 & $\begin{array}{l}11 \\
(450)\end{array}$ & $\begin{array}{l}12 \\
(9.1)\end{array}$ & $\begin{array}{l}14 \\
(16.7)\end{array}$ & $\begin{array}{l}20 \\
(42.9)\end{array}$ \\
\hline $\begin{array}{l}\text { Psychological } \\
\text { treatment }\end{array}$ & 1 & $1(0)$ & $\begin{array}{l}5 \\
(400)\end{array}$ & $\begin{array}{l}11 \\
(120)\end{array}$ & $\begin{array}{l}14 \\
(27.3)\end{array}$ & 19 & $\begin{array}{l}28 \\
(47.4)\end{array}$ & $\begin{array}{l}54 \\
(92.9)\end{array}$ & $\begin{array}{l}107 \\
(98.1)\end{array}$ & $\begin{array}{l}176 \\
(64.5)\end{array}$ & 6 & $\begin{array}{l}14 \\
(133.3)\end{array}$ & $\begin{array}{l}21 \\
(50)\end{array}$ & $\begin{array}{l}46 \\
(119)\end{array}$ & $\begin{array}{l}72 \\
(56.5)\end{array}$ \\
\hline Family-therapy & 2 & $3(50)$ & $\begin{array}{l}1 \\
(-66.7)\end{array}$ & $\begin{array}{l}4 \\
(300)\end{array}$ & $\begin{array}{l}11 \\
(175)\end{array}$ & 37 & $\begin{array}{l}87 \\
(135.1)\end{array}$ & $\begin{array}{l}108 \\
(24.1)\end{array}$ & $\begin{array}{l}183 \\
(69.4)\end{array}$ & $\begin{array}{l}235 \\
(28.4)\end{array}$ & 19 & $\begin{array}{l}49 \\
(157.9)\end{array}$ & $\begin{array}{l}42 \\
(-14.3)\end{array}$ & $\begin{array}{l}63 \\
(50)\end{array}$ & $\begin{array}{l}73 \\
(15.9)\end{array}$ \\
\hline $\begin{array}{l}\text { Group counseling/ } \\
\text { mixed counseling }\end{array}$ & 30 & $\begin{array}{l}57 \\
(90)\end{array}$ & $\begin{array}{l}113 \\
(98.2)\end{array}$ & $\begin{array}{l}189 \\
(67.3)\end{array}$ & $\begin{array}{l}219 \\
(15.9)\end{array}$ & 16 & $\begin{array}{l}35 \\
(118.8)\end{array}$ & $\begin{array}{l}49 \\
(40)\end{array}$ & $\begin{array}{l}93 \\
(89.8)\end{array}$ & $\begin{array}{l}123 \\
(32.3)\end{array}$ & 7 & $\begin{array}{l}13 \\
(85.7)\end{array}$ & $\begin{array}{l}27 \\
(107.7)\end{array}$ & $\begin{array}{l}30 \\
(11.1)\end{array}$ & $\begin{array}{l}54 \\
(80)\end{array}$ \\
\hline Mindfulness & 0 & $0(-)$ & $1(-)$ & $\begin{array}{l}12 \\
(1100)\end{array}$ & $\begin{array}{l}52 \\
(333.3)\end{array}$ & 0 & $1(-)$ & $\begin{array}{l}6 \\
(500)\end{array}$ & $\begin{array}{l}38 \\
(533.3)\end{array}$ & $\begin{array}{l}115 \\
(202.6)\end{array}$ & 0 & $1(-)$ & $\begin{array}{l}4 \\
(300)\end{array}$ & $\begin{array}{l}17 \\
(325)\end{array}$ & $\begin{array}{l}70 \\
(311.8)\end{array}$ \\
\hline Digital medicine & 0 & $0(-)$ & $2(-)$ & $\begin{array}{l}8 \\
(300)\end{array}$ & $\begin{array}{l}33 \\
(312.5)\end{array}$ & 0 & $0(-)$ & $0(-)$ & $3(-)$ & $\begin{array}{l}22 \\
(633.3)\end{array}$ & 0 & $0(-)$ & $1(-)$ & $1(0)$ & $\begin{array}{l}16 \\
(1500)\end{array}$ \\
\hline Telephony & 5 & $\begin{array}{l}3 \\
(-40)\end{array}$ & $\begin{array}{l}10 \\
(233.3)\end{array}$ & $\begin{array}{l}18 \\
(80)\end{array}$ & $\begin{array}{l}27 \\
(50)\end{array}$ & 0 & $4(-)$ & $\begin{array}{l}12 \\
(200)\end{array}$ & $\begin{array}{l}25 \\
(108.3)\end{array}$ & $\begin{array}{l}32 \\
(28)\end{array}$ & 1 & $\begin{array}{l}2 \\
(100)\end{array}$ & $\begin{array}{l}9 \\
(350)\end{array}$ & $\begin{array}{l}11 \\
(22.2)\end{array}$ & $\begin{array}{l}34 \\
(209.1)\end{array}$ \\
\hline Acupuncture & 1 & $\begin{array}{l}4 \\
(300)\end{array}$ & $\begin{array}{l}2 \\
(-50)\end{array}$ & $\begin{array}{l}10 \\
(400)\end{array}$ & $\begin{array}{l}15 \\
(50)\end{array}$ & 4 & $\begin{array}{l}9 \\
(125)\end{array}$ & $\begin{array}{l}3 \\
(-66.7)\end{array}$ & $\begin{array}{l}15 \\
(400)\end{array}$ & $\begin{array}{l}20 \\
(33.3)\end{array}$ & 4 & $\begin{array}{l}1 \\
(-75)\end{array}$ & $\begin{array}{l}4 \\
(300)\end{array}$ & $\begin{array}{l}8 \\
(100)\end{array}$ & $\begin{array}{l}11 \\
(37.5)\end{array}$ \\
\hline \multicolumn{16}{|l|}{ Pharmaceutical therapy } \\
\hline $\begin{array}{l}\text { Nicotine } \\
\text { replacement } \\
\text { therapy }\end{array}$ & 8 & $\begin{array}{l}15 \\
(87.5)\end{array}$ & $\begin{array}{l}33 \\
(120)\end{array}$ & $\begin{array}{l}53 \\
(60.6)\end{array}$ & $\begin{array}{l}64 \\
(20.8)\end{array}$ & & & & & & & & & & \\
\hline $\begin{array}{l}\text { Non-nicotine } \\
\text { medication }\end{array}$ & 1 & $\begin{array}{l}2 \\
(100)\end{array}$ & $\begin{array}{l}1 \\
(-50)\end{array}$ & $\begin{array}{l}0 \\
(-100)\end{array}$ & $0(-)$ & & & & & & & & & & \\
\hline Herbal medicine & 0 & $0(-)$ & $0(-)$ & $2(-)$ & $2(0)$ & & & & & & & & & & \\
\hline Alternative-Drug & & & & & & 14 & $\begin{array}{l}19 \\
(35.7)\end{array}$ & $\begin{array}{l}38 \\
(100)\end{array}$ & $\begin{array}{l}53 \\
(39.5)\end{array}$ & $\begin{array}{l}70 \\
(32.1)\end{array}$ & & & & & \\
\hline Buprenorphine & & & & & & 15 & $\begin{array}{l}36 \\
(140)\end{array}$ & $\begin{array}{l}107 \\
(197.2)\end{array}$ & $\begin{array}{l}223 \\
(108.4)\end{array}$ & $\begin{array}{l}328 \\
(47.1)\end{array}$ & & & & & \\
\hline \multicolumn{2}{|c|}{$\begin{array}{l}\text { Naltrexone (for drug use } \\
\text { disorder) }\end{array}$} & & & & & 21 & $\begin{array}{l}35 \\
(66.7)\end{array}$ & $\begin{array}{l}73 \\
(108.6)\end{array}$ & $\begin{array}{l}135 \\
(84.9)\end{array}$ & $\begin{array}{l}164 \\
(21.5)\end{array}$ & & & & & \\
\hline \multicolumn{2}{|l|}{ Methadone } & & & & & 170 & $\begin{array}{l}250 \\
(47.1)\end{array}$ & $\begin{array}{l}411 \\
(64.4)\end{array}$ & $\begin{array}{l}711 \\
(73)\end{array}$ & $\begin{array}{l}830 \\
(16.7)\end{array}$ & & & & & \\
\hline \multicolumn{2}{|l|}{ Herbal medicine } & & & & & 0 & $1(-)$ & $\begin{array}{l}6 \\
(500)\end{array}$ & $\begin{array}{l}5 \\
(-16.7)\end{array}$ & $8(60)$ & & & & & \\
\hline \multicolumn{2}{|l|}{ Disulfiram therapy } & & & & & & & & & & 4 & $\begin{array}{l}8 \\
(100)\end{array}$ & $\begin{array}{l}12 \\
(50)\end{array}$ & $\begin{array}{l}10 \\
(-16.7)\end{array}$ & $\begin{array}{l}18 \\
(80)\end{array}$ \\
\hline \multicolumn{2}{|l|}{$\begin{array}{l}\text { Naltrexone (for } \\
\text { Alcohol) }\end{array}$} & & & & & & & & & & 15 & $\begin{array}{l}35 \\
(133.3)\end{array}$ & $\begin{array}{l}43 \\
(22.9)\end{array}$ & $\begin{array}{l}60 \\
(39.5)\end{array}$ & $\begin{array}{l}77 \\
(28.3)\end{array}$ \\
\hline \multicolumn{2}{|l|}{$\begin{array}{l}\text { Campral/ } \\
\text { acamprosate }\end{array}$} & & & & & & & & & & 0 & $6(-)$ & $\begin{array}{l}5 \\
(-16.7)\end{array}$ & $\begin{array}{l}11 \\
(120)\end{array}$ & $\begin{array}{l}17 \\
(54.5)\end{array}$ \\
\hline
\end{tabular}


Table 3 Number of publication and the growth of publication (\%) (Continued)

\begin{tabular}{|c|c|c|c|c|c|c|c|c|c|c|c|c|c|c|c|}
\hline \multirow[b]{3}{*}{ Name of therapy } & \multicolumn{15}{|c|}{ Number of paper (Growth of publication - \%) } \\
\hline & \multicolumn{5}{|c|}{ Tobacco } & \multicolumn{5}{|l|}{ Drug } & \multicolumn{5}{|c|}{ Alcohol } \\
\hline & $\begin{array}{l}1993- \\
1997\end{array}$ & $\begin{array}{l}1998- \\
2002\end{array}$ & $\begin{array}{l}2003- \\
2007\end{array}$ & $\begin{array}{l}2008- \\
2012\end{array}$ & $\begin{array}{l}2013- \\
2017\end{array}$ & $\begin{array}{l}1993- \\
1997\end{array}$ & $\begin{array}{l}1998- \\
2002\end{array}$ & $\begin{array}{l}2003- \\
2007\end{array}$ & $\begin{array}{l}2008- \\
2012\end{array}$ & $\begin{array}{l}2013- \\
2017\end{array}$ & $\begin{array}{l}1993- \\
1997\end{array}$ & $\begin{array}{l}1998- \\
2002\end{array}$ & $\begin{array}{l}2003- \\
2007\end{array}$ & $\begin{array}{l}2008- \\
2012\end{array}$ & $\begin{array}{l}2013- \\
2017\end{array}$ \\
\hline Herbal medicine & & & & & & & & & & & 1 & $1(0)$ & $\begin{array}{l}5 \\
(400)\end{array}$ & $6(20)$ & $\begin{array}{l}3 \\
(-50)\end{array}$ \\
\hline
\end{tabular}

employment of mindfulness or digital medicine in the treatment of substance use disorder. Mindfulness and digital medicine, although confirmed to be effective, they are only trial with small sample size and have not been evaluated in a long-term research. Therefore, more research are needed to evaluate the outcomes of two treatment for substance use disorder [9]. Second, other systematic-review and meta-analysis focused the treatment of only alcohol dependence, tobacco dependence or drug use disorder. Our research using bibliometric analysis, thus, we could identify the global trend of all three kinds of substance use disorders and also highlight gaps in the scientific literature regarding contextual factors and multi-level sociobiological. Third, our findings suggest the need for international policy efforts that place priority on the development of research capacity in settings where substance use disorder is prevalent, frequently where the availability of relevant resources is simultaneously low. Some of the heavily cited papers in our study dealt with implementation science and patient outcomes. We suggest the promotion of evidence-informed policy making, health system strengthening, a renewed focus on sociobiological causes of substance use disorder, and recommend the consideration of technological transfers as potential long and short-term measures. This suggests a need for research policy that supports the examination of interventions that culturally adhere to different local contexts, specifically those that place priority on the collective when addressing substance use disorder within their communities.

This is the first bibliometric analysis of substance use disorder treatment literature. The use of bibliometric

Table 4 Research area and the Journal included the research area

\begin{tabular}{|c|c|c|c|c|c|c|}
\hline$\overline{\text { No }}$ & Research area & Total papers & Journal name & Total papers & Journal name & Total papers \\
\hline \multirow[t]{2}{*}{1} & \multirow[t]{2}{*}{ Substance Abuse } & \multirow[t]{2}{*}{4504} & $\begin{array}{l}\text { Journal of Substance Abuse } \\
\text { Treatment }\end{array}$ & 613 & Addiction & 400 \\
\hline & & & $\begin{array}{l}\text { Drug and Alcohol } \\
\text { Dependence }\end{array}$ & 591 & Addictive Behaviors & 324 \\
\hline \multirow[t]{2}{*}{2} & \multirow[t]{2}{*}{ Psychiatry } & \multirow[t]{2}{*}{3130} & $\begin{array}{l}\text { Drug and Alcohol } \\
\text { Dependence }\end{array}$ & 591 & Psychopharmacology & 197 \\
\hline & & & Addiction & 400 & Substance Use Misuse & 162 \\
\hline \multirow[t]{2}{*}{3} & \multirow[t]{2}{*}{ Psychology } & \multirow[t]{2}{*}{3041} & $\begin{array}{l}\text { Journal of Substance Abuse } \\
\text { Treatment }\end{array}$ & 613 & American Journal of Drug and Alcohol Abuse & 174 \\
\hline & & & Addictive Behaviors & 324 & journal of consulting and clinical psychology & 172 \\
\hline \multirow[t]{2}{*}{4} & \multirow[t]{2}{*}{ Pharmacology pharmacy } & \multirow[t]{2}{*}{1838} & $\begin{array}{l}\text { Journal of Substance Abuse } \\
\text { Treatment }\end{array}$ & 613 & American Journal of Drug and Alcohol Abuse & 174 \\
\hline & & & Addictive Behaviors & 324 & Journal of Consulting and Clinical Psychology & 172 \\
\hline \multirow[t]{2}{*}{5} & \multirow{2}{*}{$\begin{array}{l}\text { Public Environmental } \\
\text { Occupational Health }\end{array}$} & \multirow[t]{2}{*}{1549} & Nicotine Tobacco Research & 316 & BMC Public Health & 85 \\
\hline & & & Preventive Medicine & 92 & American Journal of Preventive Medicine & 65 \\
\hline \multirow[t]{2}{*}{6} & \multirow[t]{2}{*}{ General internal Medicine } & \multirow[t]{2}{*}{1311} & $\begin{array}{l}\text { Cochrane Database of } \\
\text { Systematic Reviews }\end{array}$ & 152 & American Journal of Preventive Medicine & 65 \\
\hline & & & Preventive Medicine & 92 & $\begin{array}{l}\text { JAMA Journal of The American Medical } \\
\text { Association }\end{array}$ & 56 \\
\hline \multirow[t]{2}{*}{7} & \multirow[t]{2}{*}{ Neurosciences Neurology } & \multirow[t]{2}{*}{1195} & Psychopharmacology & 197 & CNS Drugs & 59 \\
\hline & & & Neuropsychopharmacology & 74 & Pharmacology Biochemistry and Behavior & 57 \\
\hline \multirow[t]{2}{*}{8} & \multirow[t]{2}{*}{$\begin{array}{l}\text { Health Care Sciences } \\
\text { Services }\end{array}$} & \multirow[t]{2}{*}{676} & $\begin{array}{l}\text { Journal of Medical Internet } \\
\text { Research }\end{array}$ & 91 & Journal of General Internal Medicine & 40 \\
\hline & & & Psychiatric Services & 62 & $\begin{array}{l}\text { Journal of Behavioral Health Services } \\
\text { Research }\end{array}$ & 32 \\
\hline
\end{tabular}


Table 5 Most prolific authors

\begin{tabular}{|c|c|c|c|c|c|c|c|c|}
\hline No & Author & Affiliation and country & Total papers & Total citations & $\begin{array}{l}\text { Citations } \\
\text { per paper }\end{array}$ & h-index & $\begin{array}{l}\text { Papers in } \\
\text { collaboration }\end{array}$ & $\begin{array}{l}\text { Collaboration } \\
\text { index (signatures } \\
\text { per paper) }\end{array}$ \\
\hline 1 & Kathleen M. Carroll & $\begin{array}{l}\text { Yale University School of } \\
\text { Medicine, United States }\end{array}$ & 117 & 5878 & 50.2 & 41 & 114 & 6.4 \\
\hline 2 & Caryn Lerman & $\begin{array}{l}\text { University of Pennsylvania, } \\
\text { Abramson Cancer Center, } \\
\text { Annenberg School for } \\
\text { Communication, Department } \\
\text { of Psychiatry, United States }\end{array}$ & 87 & 4372 & 50.3 & 41 & 87 & 7.4 \\
\hline 3 & Nancy M. Petry & $\begin{array}{l}\text { University of Connecticut } \\
\text { Health Center, United States }\end{array}$ & 74 & 2504 & 33.8 & 26 & 67 & 3.8 \\
\hline 4 & Robert West & $\begin{array}{l}\text { St Georges University of } \\
\text { London, England }\end{array}$ & 72 & 3307 & 45.9 & 32 & 70 & 5.1 \\
\hline 5 & Frederick L. Altice & $\begin{array}{l}\text { Yale University, Department } \\
\text { of Internal Medicine, Infectious } \\
\text { Diseases Section, United States }\end{array}$ & 69 & 1913 & 27.7 & 27 & 69 & 6.3 \\
\hline 6 & Jon O. Ebbert & $\begin{array}{l}\text { Department of Laboratory } \\
\text { Medicine \& Pathology, Mayo } \\
\text { Clinic, United States }\end{array}$ & 65 & 1253 & 19.3 & 20 & 64 & 4.9 \\
\hline 7 & Raymond Niaura & Brown University, United States & 64 & 3276 & 51.2 & 33 & 63 & 7.8 \\
\hline 8 & Ling W & $\begin{array}{l}\text { University of Pennsylvania } \\
\text { School of Medicine, United } \\
\text { States }\end{array}$ & 63 & 2989 & 47.4 & 27 & 61 & 8.0 \\
\hline 9 & Maxine L. Stitzer & $\begin{array}{l}\text { Johns Hopkins University School } \\
\text { of Medicine, United States }\end{array}$ & 63 & 3055 & 48.5 & 30 & 63 & 6.0 \\
\hline 10 & Jasjit S Ahluwalia & $\begin{array}{l}\text { University of Kansas School of } \\
\text { Medicine and Kansas Cancer } \\
\text { Institute, United States }\end{array}$ & 62 & 2045 & 33.0 & 28 & 62 & 7.2 \\
\hline 11 & Edward V. Nunes & Columbia University, United States & 61 & 2034 & 33.3 & 25 & 61 & 8.9 \\
\hline 12 & Robert P. Schwartz & $\begin{array}{l}\text { Social Research Center, Friends } \\
\text { Research Institute, Baltimore, } \\
\text { United States }\end{array}$ & 55 & 1093 & 19.9 & 19 & 54 & 6.8 \\
\hline 13 & Timothy B. Baker & $\begin{array}{l}\text { University of Wisconsin Medical } \\
\text { School, United States }\end{array}$ & 54 & 4080 & 75.6 & 30 & 54 & 8.7 \\
\hline 14 & David A. Fiellin & $\begin{array}{l}\text { Division of Alcohol and Drug } \\
\text { Abuse, McLean Hospital, United } \\
\text { States }\end{array}$ & 53 & 1949 & 36.8 & 26 & 53 & 7.6 \\
\hline 15 & Charla Nich & $\begin{array}{l}\text { Yale University School of Medicine, } \\
\text { United States }\end{array}$ & 52 & 3223 & 62.0 & 28 & 52 & 6.9 \\
\hline 16 & Kevin E. O'grady & $\begin{array}{l}\text { University of Maryland, United } \\
\text { States }\end{array}$ & 52 & 1072 & 20.6 & 18 & 52 & 6.6 \\
\hline 17 & Thomas R. Kosten & $\begin{array}{l}\text { Yale University School of Medicine, } \\
\text { United States }\end{array}$ & 51 & 2059 & 40.4 & 25 & 51 & 5.7 \\
\hline 18 & Saul Shiffman & University of Tasmania, Australia & 50 & 3201 & 64.0 & 31 & 46 & 3.8 \\
\hline 19 & Roger D. Weiss & McLean Hospital, United States & 49 & 1151 & 23.5 & 20 & 48 & 7.8 \\
\hline 20 & Richard A. Brown & $\begin{array}{l}\text { The Warren Alpert Medical School } \\
\text { of Brown University, United States }\end{array}$ & 47 & 1750 & 37.2 & 20 & 47 & 7.5 \\
\hline 21 & J. Taylor Hays & $\begin{array}{l}\text { Nicotine Dependence Center, } \\
\text { Mayo Clinic, United States }\end{array}$ & 47 & 2404 & 51.2 & 21 & 47 & 5.7 \\
\hline 22 & Michael C. Fiore & $\begin{array}{l}\text { University of Wisconsin Medical } \\
\text { School, United States }\end{array}$ & 46 & 3682 & 80.0 & 24 & 46 & 8.1 \\
\hline 23 & Icro Maremmani & $\begin{array}{l}\text { Santa Chiara University Hospital, } \\
\text { University of Pisa, Italy }\end{array}$ & 46 & 636 & 13.8 & 12 & 46 & 7.0 \\
\hline 24 & Paul Aveyard & Psychology Department, King's & 45 & 1463 & 32.5 & 23 & 45 & 6.4 \\
\hline
\end{tabular}


Table 5 Most prolific authors (Continued)

\begin{tabular}{|c|c|c|c|c|c|c|c|c|}
\hline No & Author & Affiliation and country & Total papers & Total citations & $\begin{array}{l}\text { Citations } \\
\text { per paper }\end{array}$ & h-index & $\begin{array}{l}\text { Papers in } \\
\text { collaboration }\end{array}$ & $\begin{array}{l}\text { Collaboration } \\
\text { index (signatures } \\
\text { per paper) }\end{array}$ \\
\hline & & College London, England. & & & & & & \\
\hline 25 & Neal L. Benowitz & $\begin{array}{l}\text { Brigham and Women's Hospital, } \\
\text { Boston, United States }\end{array}$ & 45 & 2341 & 52.0 & 24 & 41 & 7.6 \\
\hline 26 & Bruce J. Rounsaville & $\begin{array}{l}\text { Yale University School of } \\
\text { Medicine, United States }\end{array}$ & 45 & 3313 & 73.6 & 28 & 45 & 6.8 \\
\hline 27 & Ricard S. Schottenfeld & $\begin{array}{l}\text { PT Foundation, United } \\
\text { States }\end{array}$ & 45 & 1428 & 31.7 & 21 & 45 & 7.2 \\
\hline 28 & John R. Hughes & $\begin{array}{l}\text { Penn State University, } \\
\text { United States }\end{array}$ & 44 & 3293 & 74.8 & 25 & 32 & 3.3 \\
\hline 29 & Rudolf H. Moos & $\begin{array}{l}\text { Department of Veterans } \\
\text { Affairs, United States }\end{array}$ & 44 & 2022 & 46.0 & 29 & 43 & 3.3 \\
\hline 30 & Rachel F. Tyndale & $\begin{array}{l}\text { University of Toronto, } \\
\text { Canada. }\end{array}$ & 44 & 1137 & 25.8 & 17 & 44 & 7.8 \\
\hline
\end{tabular}

and similar approaches, like scientometrics and informetrics, have been used to monitor the trends in other research areas. Bibliometric analyses serve as a helpful tool for research managers and policy makers when setting priorities and identifying strategies for research development and public resource allocation. Previous systematic reviews and meta-analyses of substance use disorder interventions have largely focused on specific issues and combined outcomes of different treatment options. Thus, the implications of these reviews were intended to inform clinical practice and the design of intervention programs. Meanwhile, our study provides an overarching view of the changes that have occurred within the substance use disorder research agenda over multiple decades. The study of these general trends is useful for clinicians, researchers, program managers, and policymakers having the over-trend of the global development in treatment for substance use disorder people. However, the application of those methods in a country different from one country to another, especially between developing countries and developed ones. Social, culture and environment can be the factors contributes to increase the differences in policy application. For instance, in Vietnam, alcohol is consumed in traditional national holidays such as the Lunar New Year, or weddings, housewarmings, even in funerals, and death anniversary, especially in mountainous or rural areas. Moreover, alcohol is familiar part of business transactions [48] . National legal minimum age for on- and off premise sales of alcohol is 18 and 21 in Vietnam and the U.S., respectively. Therefore, there is a need for increasing the perceptions of potential harms caused by alcohol and tighten the minimum age of alcohol using.
Government should get the priorities in investing more research in substance use disorder research and treatment, alongside the need to understandings local contexts.

The findings of this study should be viewed in light of its limitations. First, as the scope of our search on drug use was limited to opioid, drug, substance and marijuana, publications on other specific substances like Amphetamine-type stimulants (ATS) or other stimulants may not be covered, which may impact the thoroughness of our results and analysis. The breadth and comprehensiveness of our study may also be influence by the restriction on types of publications included - which consisted of only searchable peerreviewed research articles and reviews, as well as on language of publications - the selected documents for synthesis were written in English and work produced outside academic institutions that were written in local languages was excluded. As for keywords, our analysis of their occurrence and co-occurrence may not fully reflect the full content of the articles they are attached to [see Additional file 1]. However, as a bibliometric analysis of large volume of publications, a summary of keywords is a helpful proxy for the overall contents of these papers.

\section{Conclusions}

Compared to other bibliometric analysis of substance use disorder, our bibliometric analysis offers a rare and comprehensive picture of the global efforts of substance use disorder and treatment. This study with the visualization of co-occurrence term in the titles and abstracts allows researchers to track connections among clusters, which is essential in identifying the global research trend. Researchers and policy makers 


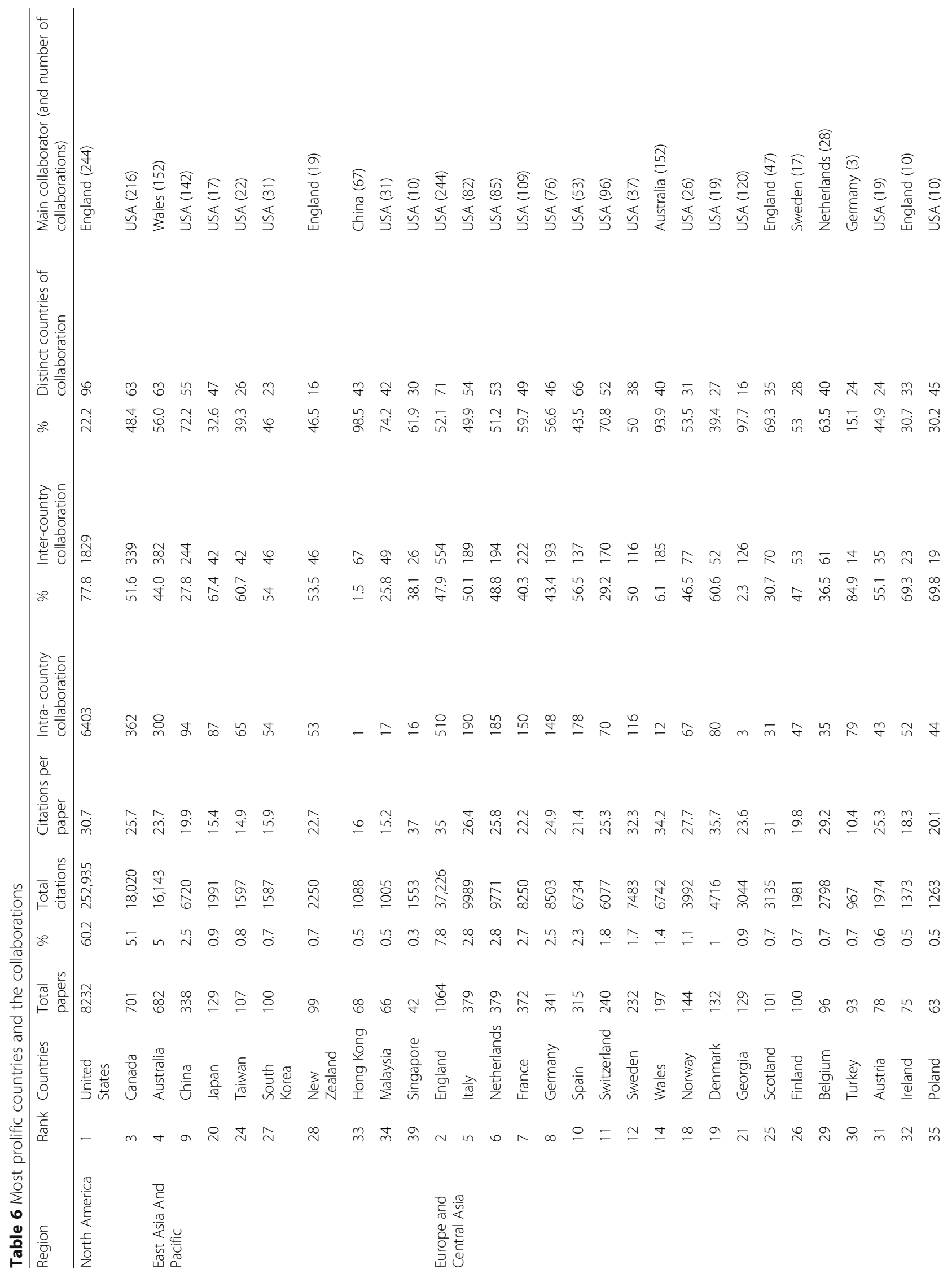




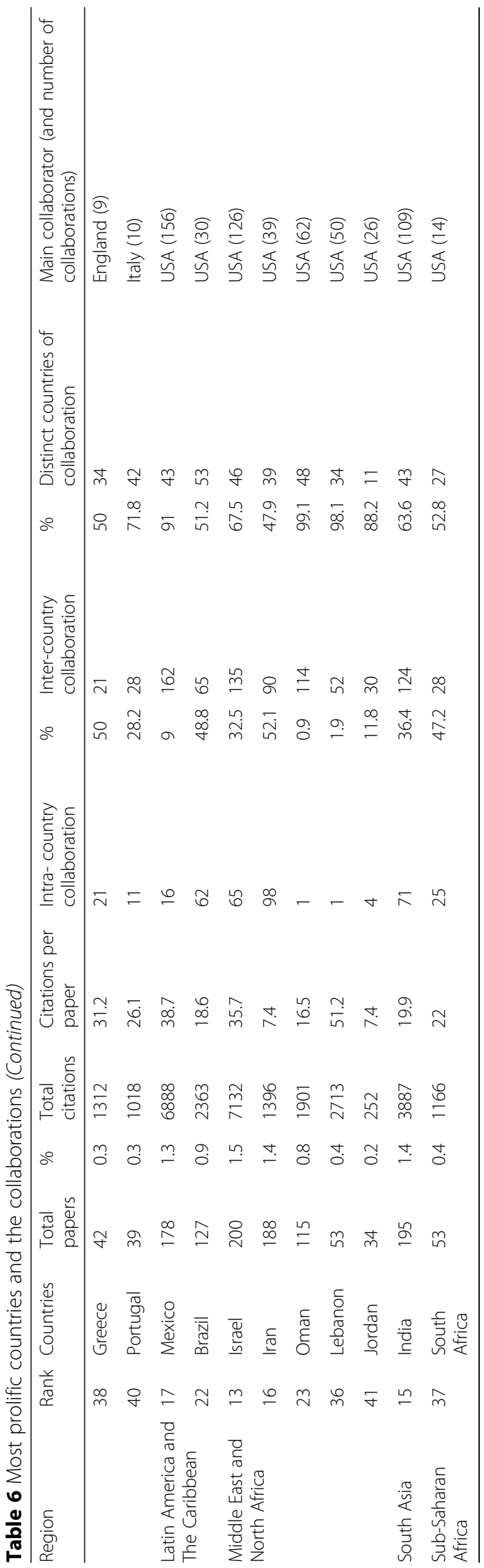




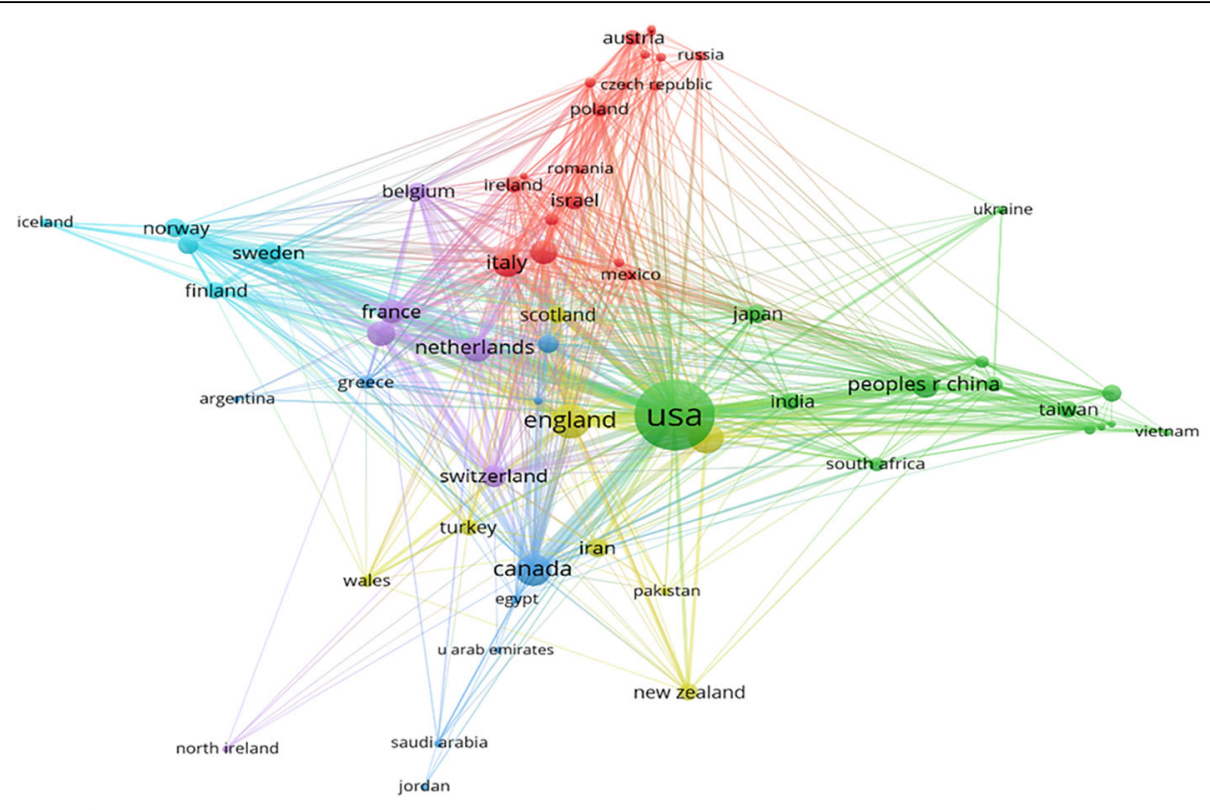

Fig. 2 A global network of the 61 most prolific countries

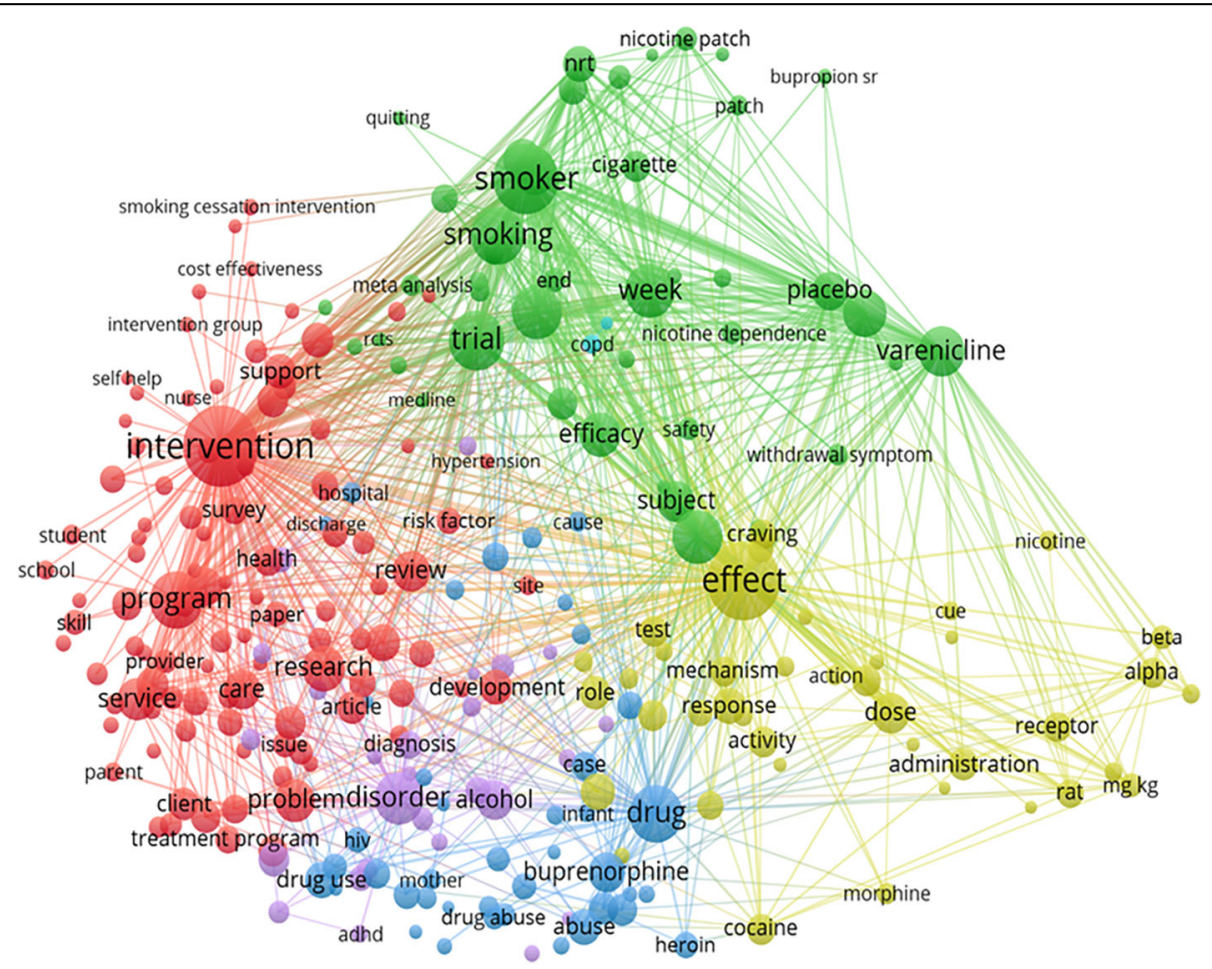

Fig. 3 Text (Title and abstract) mining 
can based on the results of this research to identify the future directions for research productions as well as the consider new therapy or prevention applying for the treatment of substance use disorder.

\section{Additional file}

Additional file 1: Figure S1. Selection of papers. Table S1. Most prolific Institution/Organizations. Table S2. Most cited papers. Figure S2. Co-Occurrence of Author's Keyword. (PDF 896 kb)

\section{Abbreviations}

DALYs: Disability-adjusted life year; UNODC: United Nations Office on Drugs and Crime; WHO: World Health Organization; YLL: Years of life lost

\section{Acknowledgements}

We would like to thank all library media specialists for their participation in the survey who supported our work in this way and helped our get results of better quality. We are also grateful to the members of our committee for their patience and support in overcoming numerous obstacles we have been facing through our research.

\section{Funding}

There was no funding for this analysis.

\section{Availability of data and materials}

The datasets used and analysed during the current study are available from the corresponding author on a reasonable request.

\section{Authors' contributions}

BXT, MM, CTN, GHH, NBN, CAL, BJH, CSHH, RCMH conceived of the study, and participated in its design and implementation and wrote the manuscript. BXT, CTN, GHH analyzed the data. BXT, GHH, CAL, BJH, RCMH helped to draft the manuscript. All authors read and approved the final manuscript.

\section{Ethics approval and consent to participate}

Not applicable

\section{Consent for publication}

Not applicable

\section{Competing interests}

The authors declare that they have no competing interests.

\section{Publisher's Note}

Springer Nature remains neutral with regard to jurisdictional claims in published maps and institutional affiliations.

\section{Author details}

'Institute for Preventive Medicine and Public Health, Hanoi Medical University, Hanoi, Vietnam. ${ }^{2}$ School of Public Health, University of Alberta, Alberta, Canada. ${ }^{3}$ Institute for Global Health Innovations, Duy Tan University, Da Nang, Vietnam. ${ }^{4}$ Center for Evidence-based Medicine, Nguyen Tat Thanh University, Ho Chi Minh City, Vietnam. ${ }^{5}$ Center for Behavioral Medicine, Nguyen Tat Thanh University, Ho Chi Minh City, Vietnam. ${ }^{6}$ Department of Psychological Medicine, National University Hospital, Singapore, Singapore. 7Department of Psychological Medicine, Yong Loo Lin School of Medicine, National University of Singapore, Singapore, Singapore. ${ }^{8}$ Bloomberg School of Public Health, Johns Hopkins University, Baltimore, MD, USA. ${ }^{9} \mathrm{Global}$ and Community Mental Health Research Group, Faculty of Social Sciences, University of Macau, Macao, SAR, People's Republic of China.

Received: 22 November 2018 Accepted: 28 March 2019 Published online: 17 May 2019

\section{References}

1. United Nations Office on Drugs and Crime V. World Drug Report 2018: Executive summary conclusions and policy implications. 2018.
2. WHO. World Drug Report 2018: Global overview of drug demand and supply. Latest trends, cross-cutting issues. p. 2018.

3. WHO. Global status report on alcohol and health 2014. Geneva; 2014.

4. World Health Organisation. WHO Global Report on Trends in Prevalence of Tobacco Smoking 2000-2025. Second edition. Geneva: World Health Organisation; 2018.

5. Whiteford HA, Degenhardt L, Rehm J, Baxter AJ, Ferrari AJ, Erskine HE, et al. Global burden of disease attributable to mental and substance use disorders: findings from the global burden of disease study 2010. Lancet (London, England). 2013;382:1575-86 Elsevier.

6. Degenhardt L, Whiteford HA, Ferrari AJ, Baxter AJ, Charlson FJ, Hall WD, et al. Global burden of disease attributable to illicit drug use and dependence: findings from the global burden of disease study 2010. Lancet. 2013;382:1564-74.

7. Murray CJL, Vos T, Lozano R, Naghavi M, Flaxman AD, Michaud C, et al. Disability-adjusted life years (DALYs) for 291 diseases and injuries in 21 regions, 1990-2010: a systematic analysis for the global burden of disease study 2010. Lancet. 2012;380:2197-223 Elsevier.

8. WHO. Disease burden and mortality estimates: Cause-Specific Mortality, 2000-2016 [Internet]. 2018. Available from: http://www.who.int/healthinfo/ global_burden_disease/GHE2016_Deaths_Global_2000_2016xxls?ua=1

9. Hay SI, Abajobir AA, Abate KH, Abbafati C, Abbas KM, Abd-Allah F, et al. Global, regional, and national disability-adjusted life-years (DALYs) for 333 diseases and injuries and healthy life expectancy (HALE) for 195 countries and territories, 1990-2016: a systematic analysis for the global burden of disease study 2016. Lancet. 2017;390:1260-344 Elsevier.

10. Jain $R$, Majumder $P$, Gupta T. Pharmacological intervention of nicotine dependence. Biomed Res Int. 2013;2013:-278392.

11. Schmelzle J, Rosser WW, Birtwhistle R. Update on pharmacologic and nonpharmacologic therapies for smoking cessation. Can Fam Phys Med De Famille Canadien. 2008;54:994-9.

12. Heydari G, Masjedi M, Ahmady AE, Leischow SJ, Lando HA, Shadmehr MB, et al. A comparative study on tobacco cessation methods: a quantitative systematic review. Int J Prev Med. 2014;5:673-8.

13. Jiloha RC. Non-Pharmacological Treatment of Alcohol Dependence. Delhi Psychiatry J. 2007:10:119-22.

14. Lu L, Liu Y, Zhu W, Shi J, Liu Y, Ling W, et al. Traditional medicine in the treatment of drug addiction. Am J Drug Alcohol Abuse. 2009:35:1-11.

15. WHO. Global strategy to reduce the harmful use of alcohol. 2010;

16. United Nations Office on Drugs and Crime (UNODC). International Standards for the Treatment of Drug Use Disorders. 2017.

17. WHO. WHO Report on the Global Tobacco Epidemic, 2015: Raising taxes on tobacco. 2015

18. WHO. Who report on the global tobacco epidemic, 2017: Monitoring tobacco use and prevention policies. 2017.

19. Wiessing L, Ferri M, Běláčková V, Carrieri P, Friedman SR, Folch $C$, et al. Monitoring quality and coverage of harm reduction services for people who use drugs: a consensus study. Harm Reduct J. 2017;14:19.

20. Baingana F, al'Absi M, Becker AE, Pringle B. Global research challenges and opportunities for mental health and substance-use disorders. Nature. 2015; 527:S172-7.

21. Ellegaard $\mathrm{O}$, Wallin JA. The bibliometric analysis of scholarly production: how great is the impact? Scientometrics. 2015;105:1809-31 Springer.

22. Sweileh WM, Zyoud SH, Al-Jabi SW, Sawalha AF. Substance use disorders in Arab countries: research activity and bibliometric analysis. Subst Abuse Treat Prev Policy 2014 [cited 2019 Feb 6];9. Available from: https://substanceabusepolicy.biomedcentral.com/articles/10.1186/1747597X-9-33.

23. Zyoud SH, Waring WS, Al-Jabi SW, Sweileh WM. Global cocaine intoxication research trends during 1975-2015: a bibliometric analysis of web of science publications. Subst Abuse Treat Prev Policy 2017 [cited 2019 Feb 6];12. Available from: http://substanceabusepolicy.biomedcentral.com/articles/10. 1186/s13011-017-0090-9

24. Valderrama-Zurián JC, Melero-Fuentes D, Aleixandre-Benavent R. Bibliographic searches for a bibliometric analysis on drug addiction. Eur Addict Res. 2015;21:31-2.

25. Khalili M, Rahimi-Movaghar A, Shadloo B, Mojtabai R, Mann K, Amin-Esmaeili M. Global scientific production on illicit drug addiction: a two-decade analysis. Eur Addict Res. 2018;24:60-70.

26. Tadmouri GO, Bissar-Tadmouri N. A major pitfall in the search strategy on PubMed. Saudi Med J. 2004;25:7-10. 
27. Falagas ME, Pitsouni El, Malietzis GA, Pappas G. Comparison of PubMed, Scopus, web of science, and Google scholar: strengths and weaknesses. FASEB J. 2008;22:338-42.

28. Kulkarni AV, Aziz B, Shams I, Busse JW. Comparisons of citations in web of science, Scopus, and Google scholar for articles published in general medical journals. JAMA. 2009:302:1092-6.

29. Substance Abuse and Mental Health Services Administration. Substance use disorders. [internet]. [cited 2018 Aug 24]. Available from: https:/www. samhsa.gov/disorders/substance-use

30. Aos S, Jim M, Miller M, Yan W. Evidence-based treatment of alcohol, drug, and mental health disorders: potential benefits, costs, and fiscal impacts for Washington state. Olympia; 2006

31. Das JK, Salam RA, Arshad A, Finkelstein Y, Bhutta ZA. Interventions for adolescent substance Abuse: an Overview of systematic reviews. The. J Adolesc Health. 2016:59:561-75 Elsevier.

32. Carney T, Myers B. Effectiveness of early interventions for substance-using adolescents: findings from a systematic review and meta-analysis. Subst Abuse Treat Prev Policy. 2012;7:25

33. Li W, Howard MO, Garland EL, McGovern P, Lazar M. Mindfulness treatment for substance misuse: a systematic review and meta-analysis. J Subst Abus Treat. 2017;75:62-96.

34. Opthof T, Wilde AAM. The Hirsch-index: a simple, new tool for the assessment of scientific output of individual scientists: the case of Dutch professors in clinical cardiology. Netherlands Heart J. 2009;17:145-54.

35. Kissin I. Scientometric assessment of drugs for chronic pain, 1979\&amp; ndash;2013: rapid growth of publications, paucity of successful drugs. J Pain Res. 2014;7:505 Dove Press.

36. Van Eck NJ, Waltman L. Text mining and visualization using VOSviewer. ISSI Newsletter:50-4.

37. van Eck NJ, Waltman L. Visualizing bibliometric networks. In: Ding Y, Rousseau R, Wolfram D, editors. Measuring scholarly impact: methods and practice [internet]. Cham: Springer International Publishing; 2014. p. 285-320. Available from: https://doi.org/10.1007/978-3-319-10377-8_13.

38. Shi J, Peng Y, Fantinato M, Chen J. A study on the author collaboration network in big data; 2017.

39. Uchtenhagen A. Substance use problems in developing countries. Bull World Health Organ. 2004:639-718.

40. Magill M, Ray LA. Cognitive-behavioral treatment with adult alcohol and illicit drug users: a meta-analysis of randomized controlled trials. J Stud Alcohol Drugs. 2009;70:516-27.

41. Hall SM, Reus VI, Munoz RF, Sees KL, Humfleet G, Hartz DT, et al, Nortriptyline and cognitive-behavioral therapy in the treatment of cigarette smoking. Arch Gen Psychiatry. 1998;55:683-90.

42. Stead LF, Perera R, Bullen C, Mant D, Lancaster T. Nicotine replacement therapy for smoking cessation. The Cochrane database of systematic reviews; 2008. p. Cd000146.

43. Zgierska A, Rabago D, Zuelsdorff M, Coe C, Miller M, Fleming M. Mindfulness meditation for alcohol relapse prevention: a feasibility pilot study. J Addict Med. 2008;2:165-73.

44. Witkiewitz K, Bowen S, Harrop EN, Douglas H, Enkema M, Sedgwick C. Mindfulness-based treatment to prevent addictive behavior relapse: theoretical models and hypothesized mechanisms of change. Subst Use Misuse. 2014;49:513-24.

45. Muench F. The promises and pitfalls of digital Technology in its Application to alcohol treatment. Alcohol Res. 2014;36:131-42.

46. Woolley R, Robinson-Garcia N, Costas R. Global research collaboration: networks and partners in South East Asia; 2017.

47. Chen K, Yao Q, Sun J, He Z, Yao L, Liu Z. International publication trends and collaboration performance of China in healthcare science and services research. Israel J Health Policy Res. 2016;5:1.

48. Lincoln M. Alcohol and drinking cultures in Vietnam: a review. Drug Alcohol Depend. 2016;159:1-8.

Ready to submit your research? Choose BMC and benefit from:

- fast, convenient online submission

- thorough peer review by experienced researchers in your field

- rapid publication on acceptance

- support for research data, including large and complex data types

- gold Open Access which fosters wider collaboration and increased citations

- maximum visibility for your research: over $100 \mathrm{M}$ website views per year

At BMC, research is always in progress.

Learn more biomedcentral.com/submissions 